\title{
Proof of the Beal Conjecture through the Fundamental Theorem of Arithmetic
}

\section{Eric S. Watson}

Abstract: This paper gives a proof of the Beal conjecture through the Fundamental Theorem of Arithmetic.

Theorem: If $A x+B y=C z$, where $A, C, x, y$ and $z$ are positive integers and $x, y, z>2$, then $A, B$ and $C$ must have a common prime factor.

\section{Proof:}

According to the Fundamental Theorem of Arithmetic, every integer greater than 1 is either a ime itself or is the product of prime numbers. This means that $A$, and $C$ are either a po itself or the product of prime numbers. Since $A$, and $C$ are raised to exponential powe grea than 2 that means that $A, B$, and $C$ are going to be the products of prime numbers then selve or the acts of other numbers that can be broken down as the product of primes. $A, C$ antrot qual to 1 because 1 is not a prime number, and 1 will always be 1 no matter of an you raise it to. This means that the lowest common prime factor that $A$ nd $C$ can, 2 .

1. Starting off with the Fundamental Theorem of Aritl etic are told that every integer greater than 1 is either a prime number itself or is the prody prime in bezs. [1]

2. Prime numbers are defined as positive integer that are divisible only by themselves and 1 only. By definition, this means that 2 is the smallest pri number. [ ] ]

3. By [1] we can say that $A$, and $C$ are either prime or or the product of prime numbers.

4. The exponents $x, y, z>2$ so the values o monents can be any positive integer as long as it is greater than or equal to 3 .

5. The exponents of a number wan times to use the number in multiplication. This means that $A x$ says to multiply $A$ vitse $x$ times, $S y$ says to multiply $B$ by itself $y$ times, and $C z$ says to multiply $C$ by itself $z$ ti es.

6. With respect to 4 minimum the $x$, and $z$ can be is 3 .

7. With respect to 2] the inimum values that $A$, or $C$ can be is 2 .

8. The comr on prime facto, hat can exist between the positive integers of $A$, and $C$ in the equation $A x+B, \quad Z z$ are $, B, C \geq 2$

9. $A$, and $C$ t have a common prime factor. According to $\mathbf{2}$ the lowest common prime factor thaca xist is

Refere

[1] http://ma voyld.wolfram.com/FundamentalTheoremofArithmetic.html

[2] http://www.mathsisfun.com/definitions/prime-number.html

[3] http://www.mathsisfun.com/definitions/exponent.html 